\title{
Constructing an Array of Anchored Single-Molecule Rotors on Gold Surfaces
}

\author{
L. Gao, ${ }^{1}$ Q. Liu, ${ }^{1}$ Y. Y. Zhang, ${ }^{1}$ N. Jiang, ${ }^{1}$ H. G. Zhang, ${ }^{1}$ Z. H. Cheng, ${ }^{1}$ W. F. Qiu, ${ }^{2}$ S. X. Du, ${ }^{1}$ Y. Q. Liu, ${ }^{2}$ \\ W. A. Hofer, ${ }^{3}$ and H.-J. Gao ${ }^{1, *}$ \\ ${ }^{1}$ Institute of Physics, Chinese Academy of Sciences, Beijing 100190, China \\ ${ }^{2}$ Institute of Chemistry, Chinese Academy of Sciences, Beijing 100190, China \\ ${ }^{3}$ Surface Science Research Centre, University of Liverpool, Liverpool L69 3BX, United Kingdom
}

(Received 10 July 2008; published 7 November 2008)

\begin{abstract}
Molecular rotors with a fixed off-center rotation axis have been observed for single tetra-tert-butyl zinc phthalocyanine molecules on an $\mathrm{Au}(111)$ surface by a scanning tunneling microscope at $\mathrm{LN}_{2}$ temperature. Experiments and first-principles calculations reveal that we introduce gold adatoms at the surface as the stable contact of the molecule to the surface. An off-center rotation axis is formed by a chemical bonding between a nitrogen atom of the molecule and a gold adatom at the surface, which gives them a welldefined contact while the molecules can have rotation-favorable configurations. Furthermore, these singlemolecule rotors self-assemble into large scale ordered arrays on $\mathrm{Au}(111)$ surfaces. A fixed rotation axis off center is an important step towards the eventual fabrication of molecular motors or generators.
\end{abstract}

DOI: 10.1103/PhysRevLett.101.197209

The motion of single atoms or molecules plays an important role in nanoscale engineering at the single atomic or molecular scale $[1,2]$. The controllability of molecular motion is critical for molecular motors [3], which may convert external energy into orchestrated motion at the molecular level [4,5]. For molecular rotors [6] a high level of control over the rotation axis and, equivalently, selfassembly on a very large scale, are the key ingredients for their integration into complex molecular machines. Previously, the reported molecular rotors on surfaces had no fixed axis on the surface [7-10]. In addition, the studies mainly focused on single molecules, while it is desirable, for eventual applications, that individual molecular rotors self-assemble into large scale ordered arrays while keeping their original functions. Here we show, using scanning tunneling microscopy (STM) [7-12], that single tetratert-butyl zinc phthalocyanine $\left((t-\mathrm{Bu})_{4}-\mathrm{ZnPc}\right)$ molecules on the reconstructed $\mathrm{Au}(111)$ surface possess a welldefined rotation axis fixed on the surface, and also, that these single-molecule rotors form large scale ordered arrays due to the reconstruction of the gold surface.

Our experiments were conducted with an Omicron MBE-LTSTM system with a base pressure below $3.0 \times 10^{-10}$ mbar. An atomically clean $\mathrm{Au}(111)$ surface was prepared by repeated cycles of $\mathrm{Ar}^{+}$sputtering and subsequent annealing, and then dosed with a minute quantity of $(t-\mathrm{Bu})_{4}-\mathrm{ZnPc}$ molecules. In our experiments, the STM tip is grounded, and the bias voltage refers to the sample voltage. All STM measurements in this study are conducted at $78 \mathrm{~K}$ if not specified. First-principles calculations were carried out based on density functional theory (DFT), a Perdew-Burke-Ernzerhof (PBE) generalized gradient approximation (GGA) for exchange-correlation energy [13], projector augmented waves (PAW) [14], and a plane wave basis set as implemented in the Vienna ab initio simulation package (VASP) [15]. A $c(5 \times 8)$ supercell was
PACS numbers: 85.85.+j, 63.20.dk, 68.37.Ef, 82.37.Gk

employed to model the isolated molecule on the gold surface. Because of numerical limitations and the size of the system, the surface Brillouin zone of the three-layer gold film was sampled with the $\Gamma$-point only. The cutoff energy for the plane waves was $400 \mathrm{eV}$. In structural relaxations, all atoms except for the bottom two gold layers were fully relaxed until the net force on every atom was smaller than $0.02 \mathrm{eV} / \AA$. According to our calculation, the gold adatom is at the hollow site.

Figure 1(a) shows an STM image of a large scale array of $(t-\mathrm{Bu})_{4}-\mathrm{ZnPc}$ molecules on $\mathrm{Au}(111)$. The molecules adsorb predominantly at the elbow positions of the surface reconstruction, typical for $\mathrm{Au}(111)$ surfaces. The $22 \times \sqrt{3}$ surface reconstruction in this case acts as an atomically precise template for the selective adsorption of $(t-\mathrm{Bu})_{4}-\mathrm{ZnPc}$ molecules on the $\mathrm{Au}(111)$ surface. A highresolution image [see Fig. 1(b)] reveals a feature of the

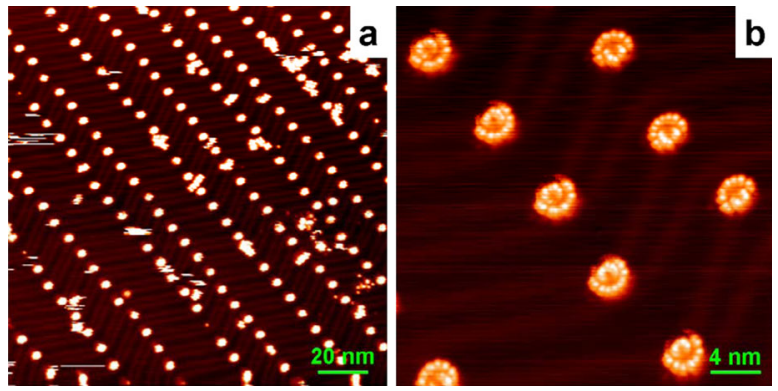

FIG. 1 (color online). (a) STM image of large scale ordered array of single $(t-\mathrm{Bu})_{4}-\mathrm{ZnPc}$ molecular rotors on the reconstructed $\mathrm{Au}(111)$ surface. (B) High-resolution STM image of single molecular rotors showing a "folding-fan" feature. The molecular rotors at two different elbow sites show different features due to the modulation by corrugation ridges. Scanning parameters: $U=-1.3 \mathrm{~V}, I=0.07 \mathrm{nA}$. Images were taken at $78 \mathrm{~K}$. 
adsorbed molecules which is reminiscent of a folding fan. The "folding-fan" feature can not be observed at $5 \mathrm{~K}$. We propose that the folding-fan feature is actually the lowfrequency image of a high-frequency molecular rotation on $\mathrm{Au}(111)$, driven by thermal energy.

To verify that the folding fan is caused by molecular instability with respect to the substrate surface, we monitored the tunneling current versus time locating the STM tip at a fixed point on the folding fan [see Fig. 2(a)], applying a constant bias voltage of $-1.8 \mathrm{~V}$ to the sample, and recording the tunneling current as a function of time. Figure 2(b) shows the recorded tunneling current within an interval of $80 \mathrm{~ms}$. The amplitude of the tunneling current oscillates with a high frequency between 0 and $5 \mathrm{nA}$. The oscillation indicates that the folding-fan feature is really due to rapid molecular motion, at the same time excluding the possibility of impurities or other artifacts.

Only one $(t-\mathrm{Bu})_{4}-\mathrm{ZnPc}$ molecule is involved for each folding-fan feature. In our experiments stationary dimers, trimers, tetramers, and larger clusters of $(t-\mathrm{Bu})_{4}-\mathrm{ZnPc}$ can be observed at $78 \mathrm{~K}$. In contrast, a stationary single $(t \text {-Bu })_{4}$-ZnPc molecule, whose STM image should be composed of four lobes, cannot be observed at $78 \mathrm{~K}$. This indicates that single molecules are not stationary but instable on the surface at this temperature. In order to observe the transition from the stationary state to the instable
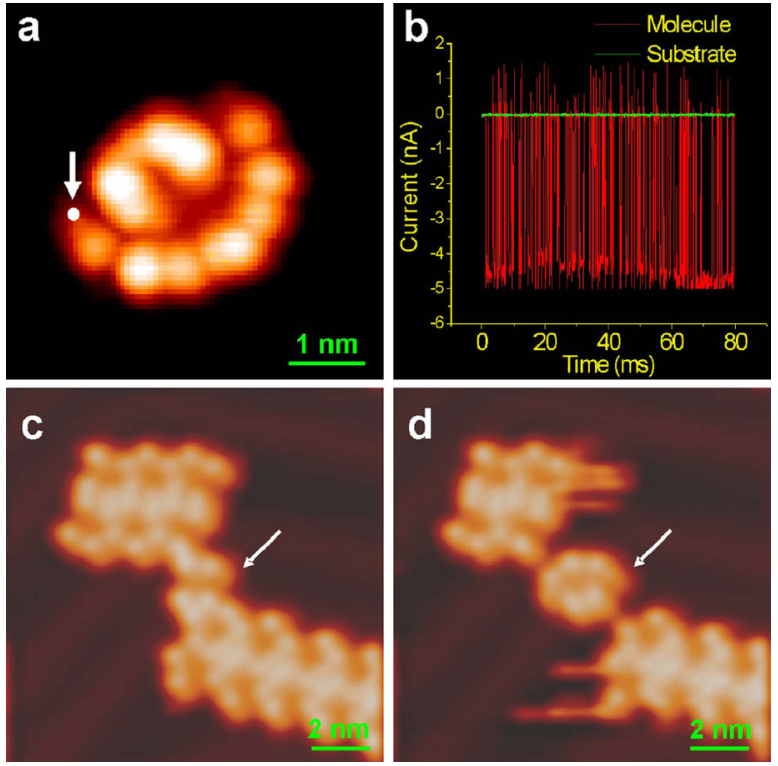

FIG. 2 (color online). (a) STM image of single $(t-\mathrm{Bu})_{4}-\mathrm{ZnPc}$ molecular rotor located at the elbow position, showing a foldingfan feature. Scanning parameters: $U=-2 \mathrm{~V}, I=0.05 \mathrm{nA}$. (b) Tunneling current versus time measured on the molecular rotor. The arrow and spot in (a) indicate the position where the $I$ - $t$ spectroscopy was measured. (c) One molecule at the elbow position, indicated by an arrow, remains stationary when it is attached to two large clusters. Scanning parameters: $U=$ $-1.4 \mathrm{~V}, I=0.1 \mathrm{nA}$. (d) The molecule starts rotating when it is isolated from the two large clusters. Scanning parameters: $U=-1.4 \mathrm{~V}, I=0.1 \mathrm{nA}$. state for single $(t-\mathrm{Bu})_{4}-\mathrm{ZnPc}$ molecule, we isolated one stationary molecule [indicated by an arrow in Fig. 2(c)], which was adsorbed at the elbow position and attached to two large molecular clusters. It was isolated from the cluster by removing its neighboring molecules with the STM tip. The molecule remained stationary when it was attached to the clusters [see Fig. 2(c)], but it became instable showing the folding-fan feature as soon as it was isolated [see Fig. 2(d)]. This manipulation demonstrates clearly that the folding-fan feature is due to the instability of a single $(t-\mathrm{Bu})_{4}-\mathrm{ZnPc}$ molecule at the elbow position on the reconstructed $\mathrm{Au}(111)$ surface.

We propose that the folding-fan feature is induced by the rotation of single $(t-\mathrm{Bu})_{4}-\mathrm{ZnPc}$ molecule. The existence of a rotation center is the prerequisite for rotation, rather than lateral diffusion along the surface at elevated temperatures. Since the center of the STM image of the molecular rotors is dark, the rotation center cannot be at the position of the tert-butyl groups which appear as bright protrusions in STM measurements. Our STM observations combined

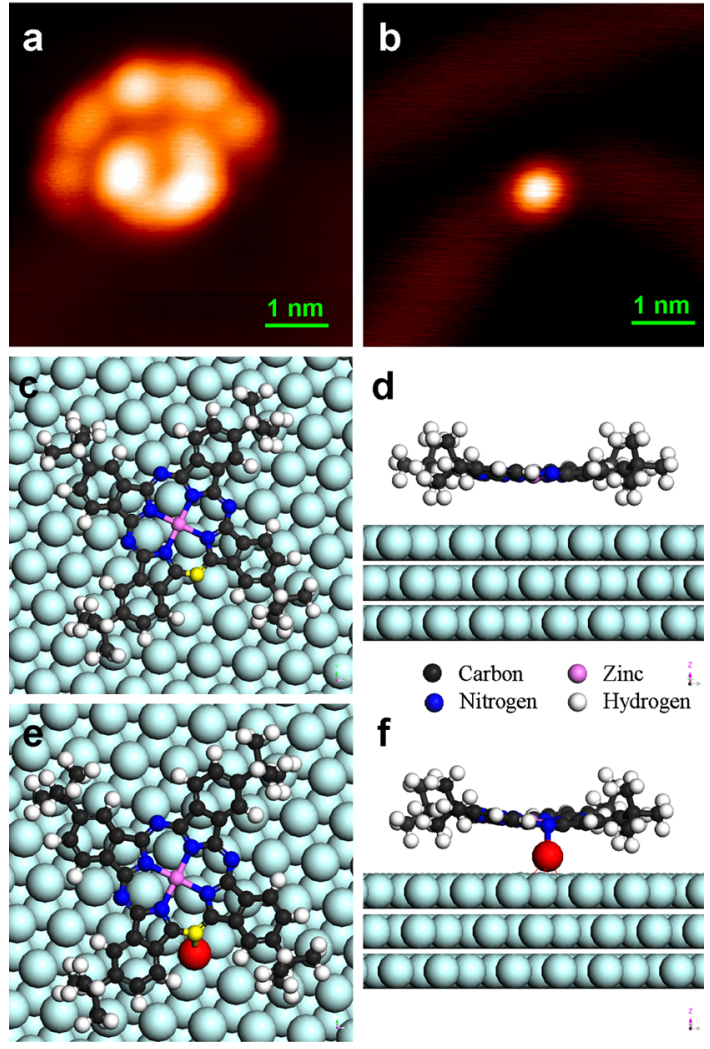

FIG. 3 (color online). (a) STM image of single molecular rotor at the elbow position. (b) Bright spot found at the rotation center after the molecule observed in (a) was removed during scanning. Scanning parameters: $U=-1.3 \mathrm{~V}, I=0.1 \mathrm{nA}$. (c) Top view and (d) side view of the optimized adsorption configuration of a $(t-\mathrm{Bu})_{4}-\mathrm{ZnPc}$ molecule on the released Au(111) surface. (e) Top view and (f) side view of the optimized configuration of a $(t-\mathrm{Bu})_{4}-\mathrm{ZnPc}$ molecule adsorbed on the $\mathrm{Au}(111)$ surface via a gold adatom. The molecular formula of $(t-\mathrm{Bu})_{4}-\mathrm{ZnPc}$ is $\mathrm{C}_{48} \mathrm{H}_{48} \mathrm{~N}_{8} \mathrm{Zn}$. 
with the first principle calculations reveal that the most likely rotation center is the gold adatom on the surface. Generally, the array of the single molecular rotors in Fig. 1 is very stable, but occasionally one molecule was removed during the scans leaving a small bright spot at the center position of the previous molecular rotor [see Figs. 3(a) and 3(b)]. This phenomenon has been observed several times. The bright spot, observed after the removal of a single molecule [see Fig. 3(b)], is most likely a gold adatom. Gold adatoms on the reconstructed gold surface are stable at $78 \mathrm{~K}$ [16], and capable of enhancing the interaction between the adsorbed molecule and the surface, forming a potential well which prevents lateral diffusion of the molecule along the surface. The gold adatoms are formed in the sample cleaning process and prefer to adsorb at the elbow sites at $78 \mathrm{~K}$. In Fig. 1(a), it is obvious that there is a bright spot at several elbow sites where no molecules adsorb. In our experiments at $78 \mathrm{~K}$, we did not find isolated single molecules that do not have the gold adatoms. This implies that the single molecules diffuse over the surface until they attach to a gold adatom or a molecular cluster.

Figures 3(c) and 3(d) show the top and side view of the optimized configuration for a single $(t-\mathrm{Bu})_{4}-\mathrm{ZnPc}$ molecule adsorbed directly on $\mathrm{Au}(111)$. Our calculations show that the distance between the zinc atom and its nearestneighbor gold atom is $4.35 \AA$, the distance between the bottom nitrogen atom [colored in yellow in Fig. 3(c)] and its nearest-neighbor gold atom $4.40 \AA$; the adsorption energy of this configuration is $219 \mathrm{meV}$. Figures 3(e) and $3(\mathrm{f})$ are the top and side view of the optimized configuration for a single $(t-\mathrm{Bu})_{4}$-ZnPc molecule adsorbed on a gold adatom. Here, our calculations show that the distance between the zinc atom and its nearest neighboring gold atom is $4.60 \AA$, the distance between the bottom nitrogen [colored in yellow in Fig. 3(e)] and the gold atom is $2.25 \AA$; the adsorption energy of this configuration in this case is $804 \mathrm{meV}$. The gold adatom enhances the molecular bonding significantly, which is most likely due to the surface dipole originating from smeared out electron charge at the position of the adatom [17]. The strong chemical bond between nitrogen and the gold adatom prevents lateral molecular diffusion along the surface, and, in particular, offers a fixed off-center axis for the rotation of single $(t-\mathrm{Bu})_{4}-\mathrm{ZnPc}$ molecules at $78 \mathrm{~K}$.

We observed that the molecular rotation depends to a large extent on the surface atomic arrangement. It is well known that the $\mathrm{Au}(111)$ surface reconstructs into a herringbone structure dividing the surface into four types of regions with different arrangements of surface atoms $[18,19]$ : face centered cubic (fcc), hexagonal close packed (hcp), corrugation ridges, and elbow sites. The regions on the surface are shown in Fig. 4(a). For the array of molecular rotors all single molecular rotors are located at the elbow sites, showing a folding-fan feature. In contrast, the STM images of the molecular rotors located in the fcc region [Fig. 4(b)], in the hcp region [Fig. 4(c)], and on the corrugation ridges [Fig. 4(d)], show "flower" features.

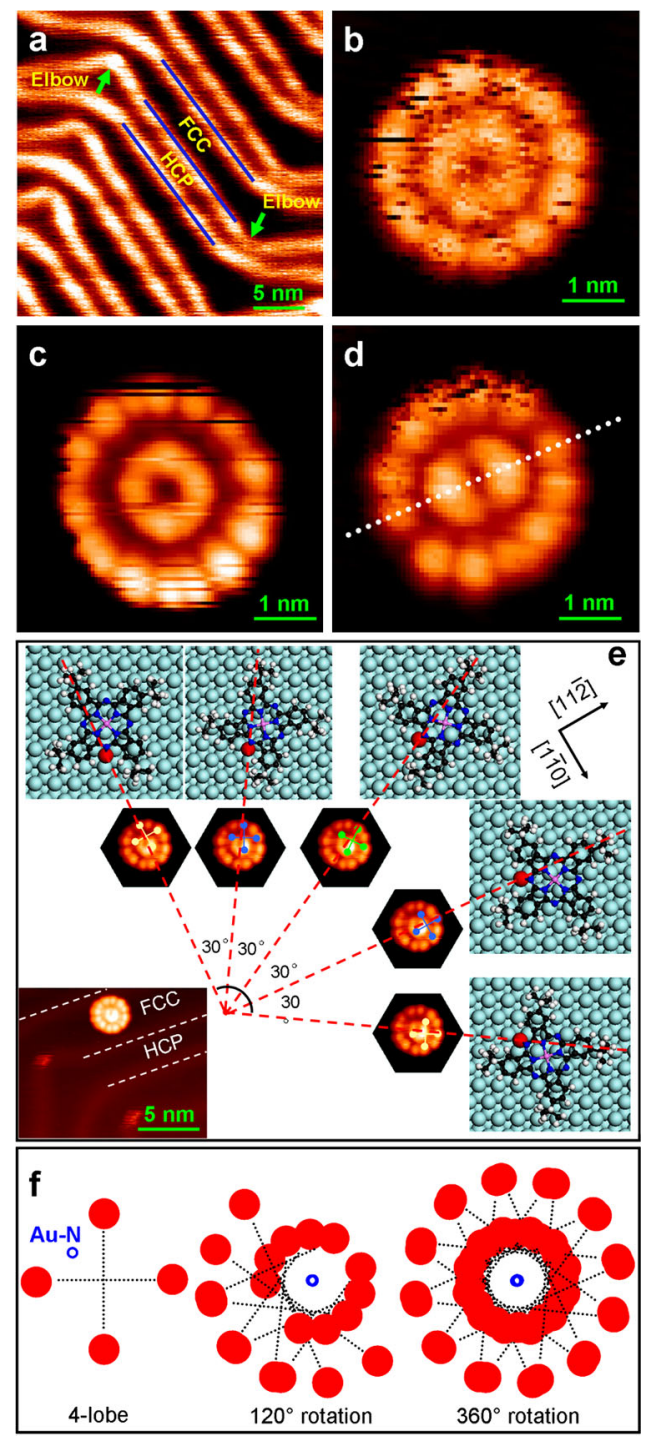

FIG. 4 (color online). (a) STM image of the reconstructed Au (111) surface. Scanning parameters: $U=-1.2 \mathrm{~V}, I=0.05 \mathrm{nA}$. (b)-(d) STM images of molecular rotors located in (b) fcc regions, (c) hcp regions, and (d) on corrugation ridges. Scanning parameters: (b) $U=-1.8 \mathrm{~V}, \quad I=0.05 \mathrm{nA}$; (c) $U=-1.0 \mathrm{~V}, I=0.05 \mathrm{nA}$; (d) $U=-1.8 \mathrm{~V}, I=0.05 \mathrm{nA}$. (e) A proposed model for the molecular rotation in the fcc region. The gold adatom is highlighted in red. The bottom-left STM image in (e) shows a typical molecular rotor in the fcc region. For the five small STM images, the cross signs represent the intermediate states for the molecular rotation, with the solid circles representing the tert-butyl groups. (f) Schematic STM images of molecular rotors with rotation angles of $120^{\circ}$ and $360^{\circ}$. The red solid circles represent the bright lobes for stationary single molecules. The blue empty circle represents the rotation center.

More specifically, STM images of molecular rotors in the fcc and hcp regions are composed of two concentric tori: twelve bright lobes form the outer torus, just like 12 "petals", while the inner torus has no obvious divisions [Figs. 4(b) and 4(c)]. The STM image of the molecular 
rotors at the corrugation ridges is composed of an outer torus, including 12 bright lobes, and two inner elliptic protrusions [Fig. 4(d)]. Both the folding-fan and flower features cannot be observed at $5 \mathrm{~K}$. Previous studies reported that molecular rotation can be controlled by chemical environment, ultraviolet or laser light, temperature, and STM manipulation. Our results reveal a novel scheme for controlling the molecular rotation with atomic precision. Here, the variation of the position of surface atoms leads to a redistribution of potential barriers for molecular rotation.

To understand the rotation of single $(t-\mathrm{Bu})_{4}-\mathrm{ZnPc}$ molecules in the fcc region we propose the following model. The bottom-left STM image in Fig. 4(e) shows the typical feature for single molecular rotor in this region. The rotation center is the gold adatom, chemically bonded to a nitrogen atom in the molecule. Calculations for several stable adsorption configurations which can be interpreted as intermediate states for the molecular rotation are shown in Fig. 4(e). The differences in adsorption energy between these stable configurations are only tens of meV. A molecule diffusing onto the gold adatom becomes stabilized [see the upper-left frame in Fig. 4(e)]. Rotating by $30^{\circ}$ around the gold adatom, the molecule reaches another stable adsorption site [see the upper-middle frame in Fig. 4(e)] with an adsorption energy reduced by $9 \mathrm{meV}$. A further rotation by $30^{\circ}$ [see the upper-right frame in Fig. 4(e)] yields an energy difference of $22 \mathrm{meV}$. Since there are 12 stable adsorption sites around the gold adatom, the molecule switches between them with high frequency under thermal excitation. Since the tert-butyl groups are imaged as the bright lobes in STM measurements, the ensuing STM image is the flower [see Fig. 4(f)]. The proposed STM image for $360^{\circ}$ rotation [see Fig. 4(f)] is in good agreement with the experimental STM image of single molecule in the fcc region [see Fig. 4(b)].

The rotation of single $(t-\mathrm{Bu})_{4}-\mathrm{ZnPc}$ molecule at the elbow sites can also be interpreted based on the model for the rotation in the fcc region. The corrugation ridges form barriers for the molecular rotation at the elbow sites. The molecular rotation is limited within an angle of $120^{\circ}$ due to the bending of the corrugation lines, which leads to the folding-fan feature. The proposed STM image for $120^{\circ}$ rotation [see Fig. 4(f)] is in good agreement with the experimental STM image of single molecule at the elbow sites [see Fig. 2(a)].

The model is in good agreement with the experimental measurements. The experimentally measured distance between the rotor center and the bright lobes on the outer torus is $1.3 \sim 1.4 \mathrm{~nm}$, in reasonable agreement with the distance between the nitrogen atom and the tert-butyl groups $(1.10 \pm 0.05 \mathrm{~nm})$, considering that the rotation center is the gold adatom which is not exactly under the nitrogen atom [see Fig. 3(e)]. Since hcp and fcc regions of the surface have the same symmetry with respect to the rotation axis, the molecular rotors in the two regions have identical STM images. The molecular rotation on the corrugation ridges can also be understood within this model, because in this case the molecular rotors are modulated by the corrugation ridges [indicated by the broken line in Fig. 4(d)]. The corrugation ridges act as the barriers for the molecular rotation at the elbow sites, leading to the folding-fan feature. There are two types of elbow sites arranging alternately along the corrugation ridges. The molecular rotation is limited in the fcc region at one type of elbow site, but in the hcp region at the other type of elbow site [see Fig. 1(b)].

In summary, we observed single molecular rotation with an off-center rotation axis fixed to surfaces, and achieved self-assembly of molecular rotors into large scale arrays. Designing the chemical bond between an atom of the molecule and an adatom on the surface we not only achieved a fixed rotation axis at the surface, but also a spin of the molecule around an off-center axis. The gold adatom, which provides the fixed rotation axis, can be used as an atomically well-defined electrode.

We thank K. Kern and S. T. Pantelides for helpful suggestions and discussion. Work at IOP was supported by grants from National Science Foundation of China, National " 863 " and "973" projects of China, the Chinese Academy of Sciences, and Supercomputing Center, CNIC, CAS. W. A. H. is supported by the Royal Society.

*hjgao@aphy.iphy.ac.cn

[1] D. M. Eigler, C.P. Lutz, and W.E. Rudge, Nature (London) 352, 600 (1991).

[2] H. Park et al., Nature (London) 407, 57 (2000).

[3] M. Schliwa, Molecular Motors (Wiley-VCH, Weinheim, Germany, 2003).

[4] W. R. Browne and B. L. Feringa, Nature Nanotech. 1, 25 (2006).

[5] E. R. Kay, D. A. Leigh, and F. Zerbetto, Angew. Chem., Int. Ed. 46, 72 (2007).

[6] G. S. Kottas et al., Chem. Rev. 105, 1281 (2005).

[7] B. C. Stipe, M. A. Rezaei, and W. Ho, Science 279, 1907 (1998).

[8] J. K. Gimzewski et al., Science 281, 531 (1998).

[9] F. Chiaravalloti et al., Nature Mater. 6, 30 (2007).

[10] L. Grill et al., Nature Nanotech. 2, 95 (2007).

[11] G. Binnig et al., Phys. Rev. Lett. 49, 57 (1982).

[12] Y. Shirai et al., Nano Lett. 5, 2330 (2005).

[13] J. P. Perdew et al., Phys. Rev. B 46, 6671 (1992).

[14] P. E. Blöchl, Phys. Rev. B 50, 17953 (1994); G. Kresse and D. Joubert, Phys. Rev. B 59, 1758 (1999).

[15] G. Kresse and J. Furthmüller, Phys. Rev. B 54, 11169 (1996); G. Kresse and J. Hafner, Phys. Rev. B 47, R558 (1993).

[16] P. Maksymovych, D. C. Sorescu, and J. T. Yates, Phys. Rev. Lett. 97, 146103 (2006).

[17] L. Limot et al., Phys. Rev. Lett. 94, 126102 (2005).

[18] C. Wöll et al., Phys. Rev. B 39, 7988 (1989).

[19] J. V. Barth et al., Phys. Rev. B 42, 9307 (1990). 